\title{
Verzeichnis der benutzten Quellen.
}

Averdunk: Die Duisburger Börtschiffahrt. Duisburg 1905.

Beck: Geschichte des Eisens. Bd. III, IV, V, VI.

van der Borght: Die wirtschaftliche Bedeutung der Rheinseeschiffahrt. Köln 1892.

Bonikowski: Der EinfluB der industricllen Kartelle auf den Handel in Deutschland. Jena 1907.

Borgius: Mannheim und die Entwickelung des südwestdeutschen Getreidehandels. I und II. Freiburg i. Br., Leipzig und Tübingen 1899.

Denkschrift des Bergbaulichen Vereins Dortmund: Die wirtschaftliche Entwickelung des niederrheinisch-westfälischen Steinkohlenbergbaues in der 2. Hälfte des 19. Jahrhunderts. Bd. I.

Duisburg am Rhein: Führer durch Duisburg. 1911.

Eckert, Chr.: Die deutsche Rheinschiffahrt im 19. Jahrhundert. Leipzig 1900.

Einecke: Eisenerzbergbau und Eisenhüttenbetrieb an der Lahn, Sieg und Dill. Jena 1907.

Führer durch die Ruhrhäfen. Ruhrort 1908.

Festschrift zur Feier der Erhebung Hamborns zur Stadt. 1911.

Gehrke: Die neucre Entwickelung des Petroleumhandels in Deutschland. Tübingen 1906.

Goecke Fedor: Der Duisburger Hafen 1826-1888.

Glückauf Nr. 34. Jahrgang 1911.

Goldstein: Die Entwickelung der deutschen Roheisenindustrie seit 1879 (Verhandlungen des Vereins zur Förderung des Gewerbefleißes 1908).

Gothein: Die Schiffahrt der deutschen Ströme (Schriften des Vereins für Sozialpolitik, Bd. 101).

Greve: Die Kanalisierung der Rubr von Wetter bis Ruhrort. Berlin 1887.

Die Gutehoffnungshütte: Festschrift zur Feier des 100jährigen Bestehens. 1910.

Handelskammerbericht Duisburg 1832-1905.

Handelskammerberichte Ruhrort 1898-1905.

Handelskammerberichte der vereinigten Handelskammern Duisburg und Ruhrort 1906-1912. 
Handelskammerberichte Mülheim-Ruhr 1840-1870.

Henz: Der Ruhrstrom und seine Schiffahrtsverhältnisse. Essen 1840.

Heymann: Die gemischten Betriebe im deutschen Großeisengewerbe. Stuttgart und Berlin 1904.

Industrielle Rundschau. Zeitschr. für Industrie und Handel. II. Jahrgang. 2. Oktober-Heft, Berlin 1899.

Jahresberichte der Rheinischen Eisenbahn 1845-1850.

Jahresberichte der Zentralkommission für die Rheinschiffahrt 18601912.

Jöhlinger: Die Praxis des Getreidegeschäftes an der Berliner Börse. Berlin 1910.

Jöhlinger: Die Technik des zheinisch-westfälischen Getreidehandels. Zeitschr. f. handelswissenschaftl. Forschung. 1906/07.

Jöhlinger: Getreidekontrakte. Schmollers Jahrbuch 1907.

Kollmann: Der deutsche Stahlwerksverband. Berlin 1905.

Marchet: Der Holzhandel Norddeutschlands. Leipzig und Wien 1908.

Mohr: Der Großbetrieb in der Getreidemüllerei. Berlin 1899.

Müpker: Denkschrift zur Hundertjahrfeier der Stadt Mülheim-Ruhr. 1908.

Nasse: Die Rhein- und Ruhrhäfen. Schriften des Vereins f. Sozialpolitik.

Nenberur: Matth. Stinnes und sein Haus. Festschrift zur Hundertjahrfeier des Hauses Stinnes.

Neumeister: Die natürlichen Grundlagen der Eisenindustrie. Schmollers Forschungen, Heft 150.

Ottmann: Die Duisburg-Ruhrorter Häfen. Denkschrift, herausgegeben im Auftrage des Ministeriums der öffentl. Arbeiten 1908.

Polster: Zur Geschichte und Entwickelung des Kohlenhandels.

Müller: Raport Consulaire sur l'année 1908-1911 (Rotterdam).

Die Rosiny-Mühlen A. G. (ohne Verlag).

Der Ruhrorter Hafen, seine Entwickelung und Bedeutung, bearbeitet durch den Wasserbauinspektor in Ruhrort, 1902.

Scherbak: Uberseeischer Maschinenexport. Berlin 1910.

Schumacher: Wanderungen der GroBindustrie in Deutschland und in den Vereinigten Staaten. Schmollers Jahrbuch 1910.

Schumacher: Die Getreidebörsen in den Vereinigten Staaten. Conrads Jahrbücher f. Nat. und Statistik 1896.

Schmid: Der süddeutsche Holzhandel. Mannheim und Leipzig 1909.

Shipping Marks on Timber. William Rider \& Son. London 1907. (Erscheint jedes Jahr.)

Sonndorfer: Die Technik des Welthandels. Wien und Leipzig 1905.

Sonndorfer: Der internationale Kohlenhandel. Wien 1910. 
Spaltowski: Die Versorgung der deutschen Hochofenindustrie mit Eisenerz. Greifswald 1909.

Stahl und Eisen 1907. Nr. 41: Die Friedrich Alfredhütte.

Stubmann: Holland und sein deutsches Hinterland in ihrem gegenseitigen Warenverkehr. Jena 1901.

Verwaltungsberichte der Stadt Duisburg 1885-1910.

Verwaltungsberichte der Gemeinde Hamborn 1900-1905.

Wiedenfeld: Der deutsche Getreidehandel. Conrads Jahrbücher für Nat.-Ök. u. Stat., 1893 u. 1894.

Wiedenfeld: Getreidehandel. Handwörterbuch der Staatswissenschaften, Bd, IV, 1909, und Wörterbuch der Volkswirtschaft (3. Aufl.), Bd. I, 1911.

Wiedenfeld: Wesen und Wert der Zentralproduktenbörsen. Leipzig, 1903.

Wiedenfeld: Die nordwesteuropäischen Welthäfen in ihrer Verkehrsund Handelsbedeutung. Berlin, 1903.

Wiedenfeld: Das Persönliche im modernen Unternehmertum. Leipzig, 1911.

Wiedenfeld: Das Rhein.-Westf. Kohlensyndikat. (Moderne Wirtschaftsgestaltungen, Heft 1) Bonn, 1912.

Zentralblatt der Bauverwaltung. Berlin, W. Ernst u. Sohn. Jahrgänge 1895, 1896, 1907.

Rheinisch-Westfälische Zeitung 1909/1910, Rhein- und Ruhrzeitung 1909/1910. Düsseldorfer General-Anzeiger 1909/1910. 